\title{
Presença de placas de ateroma calcificadas na artéria carótida: análise em radiografias panorâmicas
}

Presence of calcified atheroma plaques in the carotid artery: analysis on panoramic radiographs

Presencia de placas de ateroma calcificadas en la arteria carótida: análisis en radiografías panorámicas Íris Quintão dos SANTOS ${ }^{\mathbf{1}}$

José Jhenikártery Maia de OLIVEIRA²

Micaella Fernandes FARIAS ${ }^{2}$

Karolyne de Melo SOARES ${ }^{3}$

Murilo Quintão dos SANTOS ${ }^{4}$

Artemisa Fernanda Moura FERREIRA ${ }^{5}$

Júlio Maciel Santos de ARAÚJO ${ }^{6}$

${ }^{1}$ Cirurgiã-Dentista graduada pelo Centro Universitário de João Pessoa - UNIPÊ 58053-000 João Pessoa-PB, Brasil

${ }^{2}$ Graduando(a) do Curso de Odontologia pelo Centro Universitário de João Pessoa - UNIPÊ 58053-000 João Pessoa-PB, Brasil

${ }^{3}$ Graduanda do Curso de Odontologia pelo Centro Universitário UNIESP 58109-303, Cabedelo-PB, Brasil

${ }^{4}$ Residência em Cirurgia e Traumatologia Buco-Maxilo-Faciais pelo Hospital Universitário Lauro Wanderley HULW, Universidade Federal da Paraíba - UFPB, 58051-900, João Pessoa-PB, Brasil

${ }^{5}$ Professora Mestre do Curso de Graduação em Odontologia do Centro Universitário de João Pessoa - UNIPÊ 58053-000 João Pessoa-PB, Brasil

${ }^{6}$ Professor Doutor do Curso de Graduação em Odontologia e da Pós-Graduação (Lato sensu) em Implantodontia pelo Centro Universitário UNIESP 58109-303, Cabedelo-PB, Brasil

\section{Resumo}

Objetivo: avaliar a presença de placas de ateroma calcificadas na artéria carótida através de radiografias panorâmicas de pacientes com idade superior a 40 anos. Metodologia: trata-se de uma pesquisa de caráter descritivo, quantitativo, observacional e transversal, realizada na clínica radiográfica INTERFACE, localizada no município de João Pessoa-PB. O universo foi composto por todos os exames de radiografias panorâmicas de pacientes com idade superior a 40 anos realizados no decorrer do ano de 2015. Para tanto, a amostra foi constituída por 1000 radiografias. Utilizou-se para coleta de dados uma ficha de registro, contendo informações sobre sexo, idade, e a localização do ateroma, respeitando os critérios éticos. Os dados foram analisados e processados pelo programa SPSS (Statistical Package for the Social Sciences), versão 20.0. Resultados: A amostra apresenta radiografias com pacientes de ambos os sexos, composta por $81,60 \%$ de pacientes com ausência de placas de ateroma na artéria carótida, $11,90 \%$ com presença de placas na região direita, 4,90\% presença de placas na região esquerda e 1,60\% com presença de placas bilateralmente. De acordo com o sexo, 46,20\% do sexo feminino apresentaram ausência de placas de ateroma na artéria carótida, já o sexo masculino apenas 34,40\%. Conclusão: a prevalência de placas de ateromas calcificadas na artéria carótida foi relativamente baixa na população estudada. Ainda que a radiografia panorâmica não seja o exame de primeira escolha na identificação de placas de ateroma, foi possível observá-las na presente pesquisa, sendo assim, pode-se considerar uma ferramenta de auxílio no seu diagnóstico precoce.

Descritores: Doenças das Artérias Carótidas; Radiografia Panorâmica; Placa Aterosclerótica.

\section{Abstract}

Objective: to evaluate the presence of calcified atheroma plaques in the carotid artery using panoramic radiographs of patients over 40 years of age. Methodology: this is a descriptive, quantitative, observational and transversal research, carried out at the INTERFACE radiographic clinic, located in the city of João Pessoa-PB. The universe consisted of all panoramic radiographic examinations of patients over 40 years of age during 2015. For this purpose, the sample consisted of 1000 radiographs. A registration form was used for data collection, containing information about sex, age, and the location of the atheroma, respecting ethical criteria. The data were analyzed and processed by the SPSS program (Statistical Package for the Social Sciences), version 20.0. Results: the sample presents radiographs with patients of both sexes, comprising $81.60 \%$ of patients with no atheromatous plaques in the carotid artery, $11.90 \%$ with plaques in the right region, $4.90 \%$ with plaques in the left region and $1.60 \%$ with the presence of plaques bilaterally. According to sex, $46.20 \%$ of females presented absence of atheroma plaques in the carotid artery, while males, only $34.40 \%$. Conclusion: the prevalence of calcified atheroma plaques in the carotid artery was relatively low in the population studied. Although panoramic radiography is not the first choice exam for the identification of atheromatous plaques, it was possible to observe them in the present study, so it can be considered an aid tool in its early diagnosis.

Descriptors: Carotid Artery Diseases; Radiography, Panoramic; Plaque, Atherosclerotic.

\section{Resumen}

Objetivo: evaluar la presencia de placas de ateroma calcificadas en la arteria carótida mediante radiografías panorámicas de pacientes mayores de 40 años. Metodología: se trata de una investigación descriptiva, cuantitativa, observacional y transversal, realizada en la clínica radiográfica INTERFACE, ubicada en la ciudad de João Pessoa-PB. El universo estuvo constituido por todos los exámenes radiográficos panorámicos de pacientes mayores de 40 años durante 2015. Para ello, la muestra estuvo constituida por 1000 radiografías. Para la recogida de datos se utilizó un formulario de registro que contiene información sobre sexo, edad y localización del ateroma, respetando criterios éticos. Los datos fueron analizados y procesados por el programa SPSS (Statistical Package for the Social Sciences), versión 20.0. Resultados: la muestra presenta radiografías con pacientes de ambos sexos, constituyendo el $81,60 \%$ de pacientes sin placas de ateroma en la arteria carótida, el 11,90\% con placas en la región derecha, el $4,90 \%$ con placas en la región izquierda y el 1,60\% con presencia de placas bilateralmente. Según sexo, el $46,20 \%$ del sexo femenino no presentaba placas de ateroma en la arteria carótida, mientras que el sexo masculino solo el $34,40 \%$. Conclusión: la prevalencia de placas de ateroma calcificadas en la arteria carótida fue relativamente baja en la población estudiada. Si bien la radiografía panorámica no es el examen de primera elección para la identificación de placas de ateroma, fue posible observarlas en el presente estudio, por lo que puede considerarse una herramienta de ayuda en su diagnóstico precoz.

Descriptores: Enfermedades de las Arterias Carótidas; Radiografía Panorámica; Placa Aterosclerótica.

INTRODUÇÃO

O ateroma consiste em depósitos focais de lipídios na artéria, podendo proporcionar resposta inflamatória com proliferação fibroblástica e incrustação de sais de cálcio, produzindo graus variáveis de calcificação distrófica ${ }^{1}$. O processo aterosclerótico resulta 
em uma luz arterial estreita com diminuição do fluxo sanguíneo e do fornecimento de oxigênio causando não apenas cardiopatias coronarianas, como também doenças cerebrovasculares, se comportando como um líder de mortalidade mundial ${ }^{2}$.

$A$ aterosclerose pode levar muito tempo para dar demonstrações clínicas, ou nem mesmo chegar a produzir, portanto, é importante detectá-la precocemente. Para este fim, é necessário realizar exames vasculares a partir da terceira década de vida, especialmente em pessoas com antecedentes familiares, valorizando também os possíveis fatores de riscos da doença ${ }^{5}$, os quais são inúmeros e muitas vezes estão associados a dislipidemias, hipertensão arterial, tabagismo, sedentarismo, diabetes, entre outros ${ }^{6}$.

Alguns exames de imagem solicitados na Odontologia podem evidenciar as placas de ateroma calcificadas, logo, a avaliação radiográfica criteriosa consiste em uma importante ferramenta para a detecção precoce deste problema ${ }^{7}$. Neste contexto, a radiografia panorâmica é o exame imaginológico de rotina mais relevante, uma vez que além de ser o exame mais solicitado na prática clínica, permite a visualização de todo o complexo maxilomandibular e estruturas adjacentes, possibilitando o estudo da anatomia local bilateralmente e a detecção de algumas calcificações na região cervical $^{8-12}$.

Rotineiramente o ateroma calcificado é detectado e documentado como um achado incidental encontrado em radiografia panorâmica, a qual apresenta precisão diagnóstica de boa a excelente ${ }^{12}$.

Embora a radiografia panorâmica não seja o método padrão-ouro para o diagnóstico de placas de ateroma na artéria carótida, os cirurgiões-dentistas devem estar atentos durante a avaliação deste exame, principalmente em pacientes idosos que apresentem co-morbidades associadas à saúde geral, por estarem mais propensos a desenvolverem tal patologia ${ }^{11}$.

Desta forma, este trabalho teve como objetivo avaliar a presença de placas de ateroma calcificadas na artéria carótida através de exames radiográficos panorâmicos em pacientes com idade superior a 40 anos.

MATERIAL E MÉTODO

Esta pesquisa foi aprovada pelo Comitê de Ética em Pesquisa do Centro Universitário de João Pessoa (UNIPÊ) sob o número do parecer 1.485.508 e CAAE: 53115116.0.0000.5176.

Trata-se de um estudo documental, com abordagem quantitativa, de caráter descritivo e desenho transversal, realizado na Clínica de Radiologia INTERFACE, localizada no município de João Pessoa - PB.

O universo foi composto por todos os exames de radiografia panorâmica de pacientes com idade superior a 40 anos realizados no decorrer do ano de 2015. Para tanto, a amostra foi constituída por 1000 radiografias armazenadas no acervo documental da clínica, no ano já mencionado, selecionadas a partir do universo. Foram incluídas na amostra radiografias panorâmicas digitais de pacientes com idade superior a 40 anos com boa qualidade para diagnóstico, sendo excluídas aquelas que não apresentassem adequado grau de nitidez, densidade, contraste e resolução espacial, bem como as que possuíssem elevado nível de distorção ou algum erro de posicionamento.

A coleta de dados foi realizada através de uma ficha elaborada para este estudo pela equipe de pesquisa, que permitiu registrar os seguintes dados: sexo, idade, presença ou ausência de placas de ateroma calcificadas na artéria carótida, e em caso afirmativo, sua localização. Os dados foram analisados e processados pelo programa SPSS (statistical package for the social sciences), versão 20.0.

RESULTADOS E DISCUSSÃO

No presente estudo foram analisadas 1000 radiografias panorâmicas de pacientes com idades que variaram de 40 a 90 anos.

A média de idade dos pacientes portadores de placas ateromatosas calcificadas vistas através da avaliação das radiografias panorâmicas nesta pesquisa, foi de 63,8 anos. Este dado corrobora com os estudos de Friedlander e Lande ${ }^{14}$ e Tuñas et al. ${ }^{15}$, onde as médias de idades foram de 50 a 75 anos e 52 a 73 anos, respectivamente.

$\mathrm{Na}$ maior parte das radiografias panorâmicas analisadas, não foram observadas placas de ateroma calcificadas na artéria carótida $(81,60 \%)$. Nas radiografias onde foi constatada a alteração, $11,90 \%$ localizavam-se na região direita, $4,90 \%$ na região esquerda e $1,60 \%$ bilateralmente (Gráfico 1). Estes dados são corroborados pelo estudo de Tiller et al. ${ }^{14}$ onde foram avaliadas 824 radiografias, e foi possível observar que $91,0 \%$ mostraram ausência de calcificação na carótida. Nos casos onde a calcificação estava presente 5,5\% estava no lado direito, 2,3\% no lado esquerdo, e $1,2 \%$ em ambos os lados. Segundo Damrongsri et al. $^{15}$ as radiografias panorâmicas odontológicas de rotina têm sido relatadas para 
ser uma ferramenta útil na identificação de placas de calcificação na carótida, ou seja, os ateromas da artéria carótida e essas calcificações podem aparecer como uma radiopacidade nodular.



Grafico 1: Distribuição percentual das radiografias panorâmicas analisadas com ausência e presença de ateroma calcificado na artéria carótida. João Pessoa - PB, Brasil, 2016 (Fonte: dados da pesquisa).

Com relação ao sexo, observou-se certa homogeneidade na amostra com ligeira predominância do sexo feminino (58,2\%). Destes pacientes, $46,20 \%$ das mulheres e $34,40 \%$ dos homens não apresentaram placas de ateroma calcificadas na artéria carótida.

Somente os ateromas calcificados são visíveis nas radiografias panorâmicas, o que pode justificar sua baixa prevalência neste estudo. Vale salientar que este exame de imagem consiste em uma importante ferramenta diagnóstica onde ocasionalmente os cirurgiõesdentistas observam calcificações na região cervical, o que inclui os ateromas calcificados que aparecem como imagens radiopacas nas laterais desse exame, entre as vértebras C3 e C4. Nestes casos, o profissional deverá esclarecer o paciente sobre a importância de realizar uma investigação médica mais detalhada, sendo a ultrassonografia por Doppler colorido a ferramenta mais utilizada para confirmar a existência de aterosclerose ${ }^{16,17}$. De acordo com Christou et al. ${ }^{18}$ podem apresentase também como massas irregulares, nódulos radiopacos ou como paralelas linhas verticais radiopacas ao nível da margem inferior da terceira e a totalidade da quarta vértebra cervical, a cerca de 1,5 a 2,5 cm de distância. Essas calcificações das carótidas devem ser diferenciadas de cartilagens calcificadas presentes na área, linfonodos calcificados, processos esclerosantes, calcificação dos ligamentos estilo-hióideo e estilomandibular.

Os ateromas são encontrados principalmente na bifurcação da artéria carótida, sendo a principal causa do desenvolvimento de doenças que possam levar o paciente a óbito, além de ser a principal causa, na maioria das vezes, de acidente vascular encefálico, daí a importância do seu diagnóstico e tratamento precoce $^{21}$.

As taxas de calcificação na artéria carótida no lado esquerdo foram menores do que no lado direito para ambos os sexos, sendo observado que $7,2 \%$ das radiografias de mulheres apresentaram ateroma no lado direito e $3,60 \%$ no lado esquerdo, enquanto $4,7 \%$ dos homens apresentaram calcificação no lado direito em contraponto a $1,30 \%$ que apresentaram no lado esquerdo (Gráfico 2).

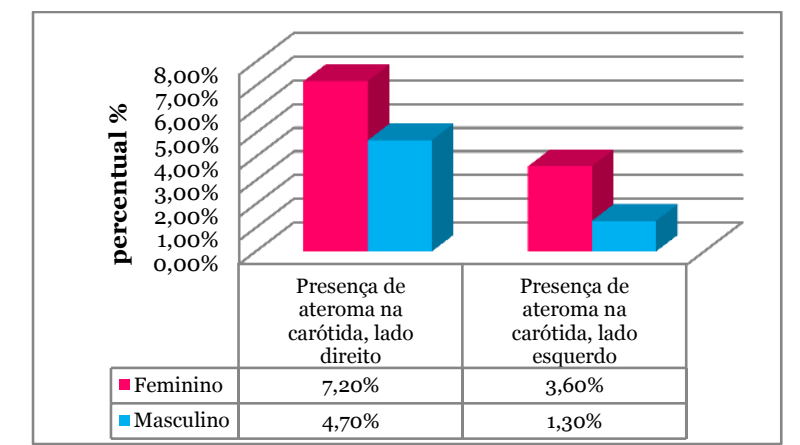

Gráfico 2: Distribuição percentual das radiografias panorâmicas analisadas de pacientes com idade superior a 40 anos de ambos os sexos com presença de placas de ateroma na artéria carótida, lados direito e esquerdo. João Pessoa -PB, Brasil, 2016 (Fonte: dados da pesquisa).

Em discordância, Tamura et al..$^{3}$ em sua pesquisa realizada no Departamento de Cirurgia Bucomaxilofacial, Faculdade de Medicina da Universidade de Mie, Japão com uma amostra de 2568 pacientes que tinham entre 50 e 70 anos, onde 1221 eram do sexo masculino e 1347 do sexo feminino, diante dos resultados, $106(4,13 \%)$ da amostra apresentaram massas nodulares suspeita de ateroma na carótida, sendo 26 homens (2,13\%) e 80 mulheres $(5,94 \%)$. Para os pacientes do sexo masculino, $88,5 \%$ estavam localizados no lado esquerdo e $38,5 \%$ estavam localizados no lado direito. Para os pacientes do sexo feminino, $77,5 \%$ estavam localizados no lado esquerdo e 30,0\% estavam localizados no lado direito. As taxas de incidência de calcificação na artéria carótida no lado esquerdo foram significativamente maior do que as taxas no lado direito para ambos os pacientes do sexo masculino e feminino.

As radiografias panorâmicas analisadas de acordo com a presença de placas de ateroma localizadas bilateralmente, em sua maior parte são compostas por pacientes do sexo feminino $(1,20 \%)$ em contraste com os $0,4 \%$ observados no sexo masculino. Estes achados se contrapões aos resultados de Uthman et al. ${ }^{22}$, os quais apresentaram maior prevalência na identificação de placas de ateroma bilateralmente no sexo masculino $(26,6 \%)$ e menor no gênero feminino $(24,4 \%)$.

\section{CONCLUSÃO}

Mediante os resultados obtidos neste estudo, conclui-se que a prevalência de placas de ateromas calcificadas na artéria carótida foi 
relativamente baixa na população estudada, existindo uma maior predileção desta alteração por pacientes do sexo feminino em todas as localizações estudadas, e o lado direito sendo o mais acometido em ambos os sexos. Desta forma, embora a radiografia panorâmica não seja o exame de primeira escolha para a identificação de placas de ateroma calcificadas na artéria carótida, neste estudo, a identificação dessas alterações nas imagens radiográficas, confirma a importância da Odontologia para o diagnóstico precoce desta condição.

\section{REFERÊNCIAS}

1. Justo MP, Pescinini Salzedas LM, Sanomiya Ikuta CR. A importância da interpretação radiográfica na detecção de calcificações na artéria carótida. Arch Health Invest. 2018;7 (Spec Iss 3):11.

2. Pereira VAC, Sousa Filho LF, Costa CHM, Beltrão RV, Almeida MSC. Imaginologia da placa ateromatosa calcificada - relato de caso. Arch Health Invest. 2018:7(Special Issue 1):38.

3. Tamura T, Inui M, Nakase M, Nakamura $S$, Okumura K, Tagawa T. Clinicostatistical study of carotid calcification on panoramic radiographs. Oral Dis. 2005;11(5):314-17.

4. Corrêa-Camacho CR, Dias-Melicio LA, Soares AMVC. Aterosclerose, uma resposta inflamatória. Arq Ciênc Saúde, 2007;14(1):41-8.

5. Senosiain-Oroquieta A, Pardo-López B, De Carlos-Villafranca F, Cobo-Plana J. Detección de placas de ateroma mediante radiografías dentales. RCOE, 2006;11(3):297-30.

6. Thelle D. Women and coronary heart disease: a review with special emplasis on some risk factors. Lipid Rev. 1990;4:34-39.

7. Roldán-Chicano R, Oñate-Sánchez RE, LópezCastaño F, Cabrerizo-Merino MC, MartínezLópez F. Panoramic radiograph as a method for detecting calcified atheroma plaques. Review of literature. Med Oral Patol Oral Cir Bucal. 2006;11(3):E261-66.

8. Fuentes Ramón, Prieto Ruth, Silva Héctor, Bornhardt Thomas, Cuevas F. Morfometría mandibular en adultos a través de uma radiografia panorâmica. Int J Morphol. 2008; 26(2):415-18.

9. Almeida-Barros RQ, Oka SCR, Holmes TSV, Cavalcanti AL, Bento PM, Godoy GP. Diagnóstico precoce de ateromas por meio de exames radiográficos rotineiros em odontologia: Considerações atuais. Odontol. Clín-Cient, 2011;10(2):129-31.

10. Freire JL, França SR, Teixeira FW, Fonteles FA, Chaves FN, Sampieri MB. Prevalence of calcification of the head and neck soft tissue diagnosed with digital panoramic radiography in Northeast Brazilian population. Minerva Stomatol. 2019;68(1):17-24.
11. Kats L, Vered M, Zlotogorski-Hurvitz A, Harpaz I. Atherosclerotic carotid plaque on panoramic radiographs: neural network detection. Int $\mathrm{J}$ Comput Dent. 2019;22(2):163-69.

12. Schroder AGD, de Araujo CM, Guariza-Filho O, Flores-Mir C, de Luca Canto G, Porporatti AL. Diagnostic accuracy of panoramic radiography in the detection of calcified carotid artery atheroma: a meta-analysis. Clin Oral Investig. 2019;23(5):2021-40.

13. Atalay Y, Asutay F, Agacayak KS, Koparal M, Adali $F$, Gulsun B. Evaluation of calcified carotid atheroma on panoramic radiographs and Doppler ultrasonography in an older population. Clin Interv Aging. 2015;10:1121-29.

14. Friedlander $\mathrm{AH}$, Lande $\mathrm{A}$. Panoramic radiographic identification of carotid arterial plaques. Oral Surg Oral Med Oral Pathol. 1981; 52(1):102-4.

15. Inger Teixeira de Campos Tuñas Eduardo José da Costa Santos Leonardo de Mello de Veiga Luiz Fernando Deluiz Sérgio de Carvalho Weyne Tuñas ITC et al. Ateromas de carótida nas panorâmicas: como o clínico pode identificar?. Rev. Bras. Odontol. 2012;69(2): 203-6.

16. Tiller R, Bengel W, Rinke S, Ziebolz D. Association between carotid area calcifications and periodontal risk: a cross sectional study of panoramic radiographic findings. BMC Cardiovasc Disord. 2011;11:67.

17.Pornprasertsuk-Damrongsri S, Virayavanich W, Thanakun S, Siriwongpairat $P$, Amaekchok P, Khovidhunkit W. The prevalence of carotid artery calcifications detected on panoramic radiographs in patients with metabolic syndrome. Oral Surg Oral Med Oral Pathol Oral Radiol Endod. 2009;108(4):e57-62.

18. Guimarães GO, Oliveira IE, Nobile Junior D, Messina Calderón JC, Saddy MS. Radiografia panorâmica: identificação de pacientes susceptíveis ao acidente vascular cerebral por meio da detecção de ateromas na bifurcação da carótida. Conscientia e Saúde. 2005;4: 97- 104.

19. Romano-Sousa CM, Krejci L, Medeiros FMMa, Graciosa-Filho RG, Martins MFF, Guedes VN et al. Diagnostic agreement between panoramic radiographs and color doppler images of carotid atheroma. J Appl Oral Sci. 2009;17(1):45-8.

20. Christou $P$, Leemann $B$, Schimmel M, Kiliaridis $S$, Müller F. Carotid artery calcification in ischemic stroke patients detected in standard dental panoramic radiographs - a preliminary study. Adv Med Sci. 2010;55(1):26-31.

21. Horsley SH, Beckstrom B, Clark SJ, Scheetz JP, Khan Z, Farman AG. Prevalence of carotid and pulp calcifications: a correlation using 
digital panoramic radiographs. Int $\mathrm{J}$ Comput Assist Radiol Surg. 2009;4(2):169-73.

22. Uthman AT, Al-Saffar AB. Prevalence in digital panoramic radiographs of carotid area calcification among Iraqi individuals with strokerelated disease. Oral Surg Oral Med Oral Pathol Oral Radiol Endod. 2008;105(4):e68-73.

\section{CONFLITO DE INTERESSES}

Os autores declaram não haver conflitos de interesse

AUTOR PARA CORRESPONDÊNCIA

José Jhenikártery Maia de Oliveira

Centro Universitário de João Pessoa (UNIPÊ) -

Rodovia BR-230, km 22, S/N - Água Fria

58053-000 João Pessoa - PB, Brasil

Telefone: +55 (83) 9 9696-0759

E-mail: jhenikarterymaia@gmail.com

Submetido em 24/09/2020

Aceito em 21/11/2020 